\title{
BMJ Open Efficacy of a personalised pelvic floor muscle training programme on urinary incontinence after radical prostatectomy (MaTchUP): protocol for a randomised controlled trial
}

\author{
Paul Hodges, ${ }^{1}$ Ryan Stafford, ${ }^{1}$ Geoff D Coughlin, ${ }^{2,3}$ Jessica Kasza, ${ }^{4}$ \\ James Ashton-Miller, ${ }^{5}$ Anne P Cameron, ${ }^{5}$ Luke Connelly, ${ }^{6,7}$ Leanne M Hall ${ }^{1}$
}

To cite: Hodges P, Stafford R, Coughlin GD, et al. Efficacy of a personalised pelvic floor muscle training programme on urinary incontinence after radical prostatectomy (MaTchUP): protocol for a randomised controlled trial. BMJ Open 2019;9:e028288. doi:10.1136/ bmjopen-2018-028288

- Prepublication history for this paper is available online. To view these files, please visit the journal online (http://dx.doi org/10.1136/bmjopen-2018028288).

Received 30 November 2018 Revised 18 March 2019 Accepted 26 March 2019
Check for updates

(C) Author(s) (or their employer(s)) 2019. Re-use permitted under CC BY-NC. No commercial re-use. See rights and permissions. Published by BMJ.

For numbered affiliations see end of article.

Correspondence to

Dr Paul Hodges;

p.hodges@uq.edu.au

\section{ABSTRACT}

Introduction Prostate cancer is the most common cancer in men. Prostatectomy is the most common treatment. Morbidity from prostatectomy is high- $80 \%$ of men experience urinary incontinence which negatively impacts the quality of life. Postsurgical pelvic floor muscle training is commonly prescribed but recent systematic reviews found no evidence of efficacy. We propose a new treatment that commences preoperatively and targets functional training of specific pelvic floor muscles that contribute to urinary continence. Assessment and biofeedback using transperineal ultrasound imaging assists in training. This will be compared against conventional training (maximal pelvic floor muscle contraction assessed by digital rectal examination) and no training. Embedded physiological studies will allow the investigation of moderation and mediation of the treatment effect on the outcomes. Methods and analysis This randomised clinical trial will include 363 men scheduled to undergo radical prostatectomy for prostate cancer. Participants will be randomised into urethral training, conventional training and no training groups. Clinical data will be collected at baseline (1-2 weeks presurgery) and postsurgery after catheter removal, weekly to 3 months (primary endpoint) and monthly to 12 months. Outcomes include 24-hour pad weight test (primary), incontinence, quality of life and cost-effectiveness data. Neuromuscular control measures of pelvic floor muscles will be measured at baseline, postsurgery, 6 weeks, 3 and 12 months. Study assessors and statisticians will be blinded to the group allocation. Ethics and dissemination This study is registered with the Australian New Zealand Clinical Trials Registry and has ethical approval from the university and host hospital ethics committees. Trial outcomes will be shared via national/international conference presentations and peerreviewed journal publications.

Trial registration number ACTRN12617000788370; Preresults.

\section{INTRODUCTION}

Prostate cancer is the most common non-cutaneous cancer in men in Australia and

\section{Strengths and limitations of this study}

- Uses randomised design in a clearly defined population.

- Tests an innovative intervention designed to target mechanisms of urinary continence in men that is based on recent physiological data of mechanisms of continence and incontinence in men.

- Uses individualised care based on assessment using new transperineal ultrasound imaging methods to study pelvic floor muscle function.

- Includes investigation of mediation and moderation of the treatment effect by pelvic floor muscle neuromuscular control variables.

- Possible limitations are adherence to the comprehensive home programme and the burden of the extensive follow-up data collection.

internationally (one in seven men) and the second most common cause of cancer death. ${ }^{1}$ Radical prostatectomy (prostate removal to prevent metastasis) is a common treatment. The good news is that prostate cancer has very high 5-year survival-95\%. ${ }^{1}$ The bad news is that morbidity is high-almost $80 \%$ of men experience incontinence after prostatectomy (postprostatectomy incontinence $[\mathrm{PPI}]){ }^{2}$ and many are incontinent beyond 12 months $^{2}$. PPI has been identified as the major determinant of quality of life for these men, and many live for many years with ongoing major cost (up to $33 \%$ use incontinence products ${ }^{3}$ ) and social isolation. ${ }^{45}$ Introduction of robotic prostatectomy has not reduced PPI. ${ }^{6}$ Effective methods to reduce PPI are a priority.

Pelvic floor muscle training (PFMT) to enhance muscular control of urinary continence after prostatectomy is logical. Although the efficacy of PFMT in women stress urinary incontinence has class 1 evidence, $^{7}$ early 
optimistic outcomes for $\mathrm{PPI}^{8}$ are superseded by systematic review evidence of no efficacy in men. ${ }^{9}$ Recent physiological research using innovative ultrasound imaging ${ }^{10}{ }^{11}$ and electromyography methods ${ }^{12}$ suggests that conventional PFMT programmes, which involves repeated maximal contractions assessed by digital rectal examination and commenced after surgery, ${ }^{13}$ fail to consider the mechanisms of incontinence after prostatectomy, are unlikely to target the muscles that control urinary continence, do not target the aspects of function that need to be trained and start too late.

Urinary continence in men depends on the contributions from smooth muscle of the urethra and urethral constriction generated by contraction of three striated muscles: the striated urethral sphincter (SUS); puborectalis/pubovisceralis (PR) and bulbocavernosus (BC). ${ }^{10}$ These striated muscles maintain gentle activation during urine storage ${ }^{1214}$ with additional activation when continence is challenged by elevated intra-abdominal pressure such as coughing ${ }^{15}$ or postural tasks. ${ }^{14}$ Radical prostatectomy inherently removes the prostatic segment of the urethra, and its smooth muscle (called the internal sphincter), and may remove/damage the SUS muscle ${ }^{16}$ or its innervation. ${ }^{17}$ Surgery may also affect the smooth muscle of the bladder neck, ${ }^{17}$ as well as bladder contractility ${ }^{18}$ and compliance, ${ }^{16}$ contributing to overactivity of the detrusor muscle. ${ }^{16} 18$ Recovery of continence after prostatectomy is likely to require: enhanced function of SUS (and other striated muscles) to compensate for the reduced smooth muscle (which would require capacity for low-intensity sustained contraction in addition to strong contraction); compensation by the PR and BC if SUS is affected by surgery and training of the bladder to hold volume. Recent work has highlighted that persistent PPI is associated with impaired shortening of the SUS and BC and descent rather than elevation of the bladder neck (explained by the failure of PR to prevent depression from excessive abdominal pressure) during voluntary activation, but this varies between men. ${ }^{10}$ Digital rectal examination used for assessment and feedback in most previous trials of PFMT for incontinence after prostatectomy ${ }^{19}$ provides information on anal sphincter and PR contraction but cannot provide information on the SUS and BC. Transperineal ultrasound imaging provides a non-invasive and validated ${ }^{20}$ method to evaluate and provide feedback of PR, SUS and BC, simultaneously.

Supported by this physiological evidence and pilot clinical data, we predict that by implementation of a PFMT programme that targets the muscles that control urethral pressure (particularly SUS) and compensates for tissues removed during surgery, in a manner that matches the individual needs of each man, and trains incorporation of pelvic floor muscle activation into functional tasks (rather than a training programme limited to repeated maximal voluntary contractions), we can achieve superior outcomes with substantial impact on quality of life after prostatectomy.

\section{Aim}

In this trial we aim to:

1. Determine whether PFMT that involves individualised functional training of neuromuscular (NM) control of striated muscles that constrict the urethra (urethral training) achieves more rapid continence recovery after radical prostatectomy than a PFMT programme that involves brief strong contractions of muscles around the anus that are not specific for urinary continence (conventional training) or no training.

2. Test whether the quality of NM control of striated muscles that constrict the urethra at baseline (prior to surgery) moderates the relative efficacy of urethral training compared with conventional training or no training.

3. Determine whether a change in NM control of striated muscles that constrict the urethra mediates the recovery of urinary continence.

4. Compare the cost-effectiveness of the training programmes.

\section{METHODS AND ANALYSIS \\ Study design}

This manuscript describes a research protocol for the 'personalised pelvic floor Muscle Training for Urinary incontinence after Prostatectomy' (MaTchUP) randomised controlled trial (RCT). This study is a prospectively registered, RCT. Participants will be randomised into either urethral training, conventional training or no training. This protocol has been developed in accordance with Standard Protocol Items: Recommendations for Interventional Trials. ${ }^{21}$

\section{Participant recruitment}

Men scheduled to undergo radical prostatectomy (open or robotic) for prostate cancer at the Wesley and Princess Alexandra Hospitals (Brisbane, Queensland) will be invited to participate via an information pamphlet provided by administrative staff during a presurgical consultation with their treating urologist.

\section{Participants}

To be eligible, participants must meet the following criteria:

Inclusion criteria:

- Scheduled to undergo radical prostatectomy for prostate cancer.

- 30-70 years of age.

- Able to attend assessment/rehabilitation sessions in Brisbane.

- Able to understand English.

Exclusion criteria:

- Urinary incontinence prior to surgery.

- Previous prostate surgery.

- Assessment/training of the pelvic floor muscles in the preceding 6 months.

- Scheduled to undergo or had previously undergone radiation therapy for prostate cancer. 


\section{Study treatments}

Volunteers will be screened via an online form and phone interview and then scheduled to undergo a baseline assessment in the laboratory 1-2 weeks prior to surgery. After baseline assessment, participants will be randomised into one of three groups: conventional training, urethral training or no training.

Randomisation will be in permuted blocks of size 4-8, stratified by surgeon and baseline NM control of striated muscles that constrict the urethra with patients dichotomised as good (or poor) by ability to achieve both $\geq 4.1 \mathrm{~mm}$ of SUS displacement and $\geq 2.4 \mathrm{~mm}$ of PR displacement as assessed with transperineal ultrasound imaging (see below; RE Stafford et al, unpublished data, 2018). Group allocation will be determined using an automatic randomisation schedule developed by an independent statistician and integrated into the Research Electronic Data Capture (REDCap) data administration system (see below) to ensure concealed allocation.

Participants randomly allocated to the groups receiving treatment will attend up to 10 treatment sessions supervised by a physiotherapist. All participants will attend an initial session 1-2 weeks before surgery (after the baseline assessment). At this session, participants in the no training group will undergo no assessment or training and will not attend further sessions. Participants allocated to one of the two treatment groups will be assessed by the physiotherapist at the preoperative session according to their allocated exercise programme and taught the initial training exercise to commence prior to surgery. After surgery, participants in the exercise groups will attend up to nine sessions, 1 week apart, commencing after catheter removal ( $\sim 2$ weeks postsurgery) and continued until continence is achieved (using the definition below) or until 10 sessions have been used. Exercise programmes will be documented according to the Consensus on Exercise Reporting Template guidelines ${ }^{22}$ which has been adapted specifically for recording PFMT programmes by Hall et al. ${ }^{23}$

\section{Conventional training}

The conventional PFMT is focused on the repeated maximal contraction of the muscles around the anus and follows the principles of the programme ${ }^{13}$ used in the largest previous RCT for men with PPI. ${ }^{24}$ Training commences with an assessment of muscle activation (digital rectal examination or anal surface electromyography [EMG]). Participants perform 3s maximal contractions in lying, sitting and standing, two times per day, and also before activities such as coughing, lifting, rising from sitting. Daily home exercises are encouraged and monitored by a physiotherapist. Training progresses by increasing the duration of contractions up to $10 \mathrm{~s}$.

\section{Urethral training}

Urethral training is an individualised PFMT programme focused on the striated pelvic floor muscles that constrict the urethra (SUS, PR, BC) with progression according to a decision tool developed with a clinical advisory committee. Exercise relies on the principles of motor learning, skill training and exercise physiology. The training uses transperineal ultrasound imaging for assessment of pelvic floor muscle activation during voluntary contraction, coughing and a $60 \mathrm{~s}$ maximal contraction ${ }^{11} 1520$ to guide treatment tailoring. Transperineal ultrasound imaging is also used for biofeedback at each physiotherapy session. Urethral training commences with skill acquisition of the optimal pattern of pelvic floor muscle activation to increase urethral pressure and avoidance of excessive abdominal muscle contraction. Initial training focuses on SUS but with tailoring to include the other muscles based on the assessment. Progression includes: training for activation of pelvic floor muscles in functional tasks; bladder training to increase holding capacity; sustained holding to enhance adaptation of striated muscles to provide ongoing maintenance of continence; training of ballistic efforts for episodes of increased bladder pressure (lifting, coughing and so on) and high-performance training including strength training to prepare for demanding tasks. Daily home exercise to practice tasks taught in each session will be encouraged and monitored by a physiotherapist.

\section{No training}

Participants allocated to no training will attend a preoperative session with a physiotherapist during which they will receive standard written education material (similar to the online resources readily available from prostate cancer support groups) and education about how to perform the outcome measures.

Participants in all groups will be requested to refrain from seeking additional treatment until the primary endpoint at 3 months. Any treatment sought by participants will be recorded.

Physiotherapists will be trained to apply one treatment only. They will have prior experience with management of incontinence and will undergo sufficient training to ensure competence. Therapists providing urethral training will receive comprehensive training in transperineal ultrasound imaging. Competence of therapists will be formally assessed and treatment fidelity evaluated by observation during a subset of sessions by a researcher to document adherence to the protocol.

\section{Data collection}

All data will be collected online using REDCap. Participants will complete an online questionnaire at baseline to provide demographic data including date of birth, height, weight, employment status, marital status, education level, smoker status and comorbidities. Prostate volume, Gleason score, surgery date, surgery type, surgery complications, date of catheter removal and adjunct treatments will also be recorded.

Primary and secondary data (except neuromuscular control measures) will be recorded with the online system according to the schedule outlined in table 1 . The primary endpoint at 3 months was selected as qualitative research highlights that rapid/complete recovery of continence is a priority for men ${ }^{25}$ as long periods of incontinence have 


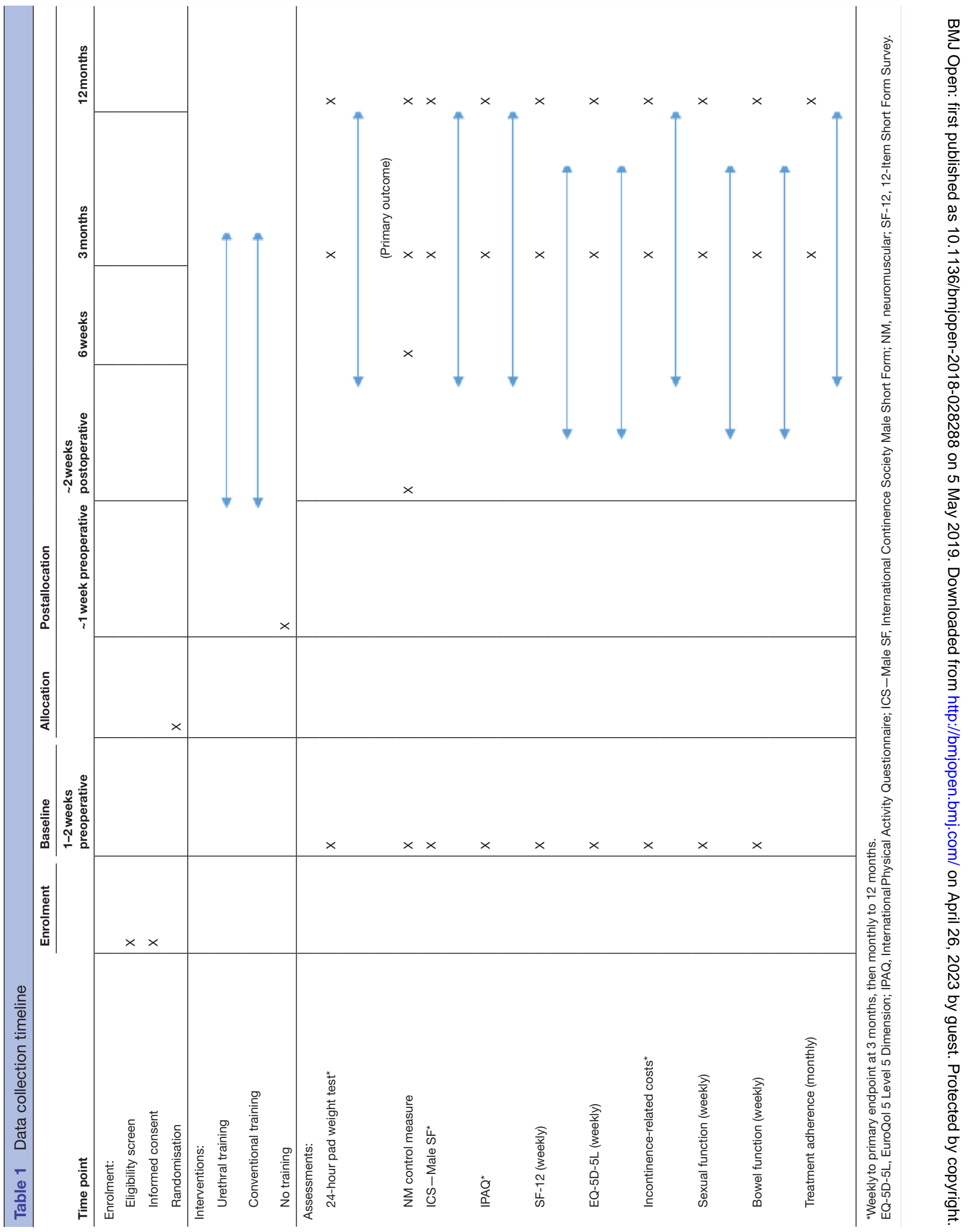


a major impact on quality of life, ${ }^{26}$ and it was considered unethical to withhold treatment from men allocated to no training for more than 3 months if they continue to experience incontinence.

For secondary outcome measures of NM control, participants will attend laboratory sessions at baseline (prior to randomisation for identification of NM control parameters used for stratification and as moderator of treatment efficacy), $\sim 2$ weeks postoperative after catheter removal (for secondary analysis of postoperative NM control as a moderator of treatment efficacy), 6 weeks (for intermediate measurement of NM control to judge improvement in NM control as a potential causal mediator of outcome) and 12 months (for secondary analysis of long-term outcome of intervention) (table 1).

Secondary outcomes (continence [continence questionnaire and 24-hour pad weight test], physical activity, quality of life, incontinence-related costs and sexual function and bowel function) will be entered into the online data management system weekly until the primary endpoint at 3 months. All secondary outcomes will also be collected at 12 months. All men will continue to complete the continence (continence questionnaire and 24-hour pad weight test), physical activity and incontinence-related costs data monthly until 12 months. Men will be prompted to complete this information via their preferred method (short message service [SMS], telephone or email).

\section{Treatment adherence}

Adherence to exercise will be encouraged by the treating physiotherapist and monitored using an online questionnaire. Physiotherapists will be trained to promote adherence to the programme using principles of behaviour change including identification of barriers, cognitive analysis, prioritisation and action planning. Participants will be prompted monthly via their preferred method (SMS, telephone or email) to input data of home exercise performance. This was selected rather than weekly measurement to avoid excessive prompting of the no treatment group.

\section{Blinding}

Assessors and statisticians will be blinded to group allocation. It will not be possible to blind the participant or treating therapist to the treatment, but the patients will be blinded to the hypotheses or details of treatments applied to other groups. Prior to randomisation, participants will be informed that systematic reviews show uncertain evidence of benefit from PFMT (note that all men, including the no treatment group, will receive written information about PFMT).

\section{Outcome measures}

Primary outcome

The primary outcome measure will be the 24-hour pad weight test. It will be assessed at baseline and 3 months. Continence is defined as a loss of $<2 \mathrm{~g}$ of urine. ${ }^{8}$ The day before laboratory testing, the SMS service will remind men to preweigh and retain all pads used for the next 24 hours in sealed plastic bags and weigh them using provided scales. Data will be provided to the research staff at the laboratory session. The dichotomous classification of continence will be the primary outcome.

\section{Secondary outcomes}

Ultrasound measures of NM control of urethral pressure: NM control of pelvic floor muscles will be assessed at baseline, postsurgery, 6 weeks, 3 months and 12 months during: (1) voluntary contraction of pelvic floor muscles; (2) cough according to a protocol described by Stafford et $\mathrm{al}^{11}$; (3) maximal voluntary contraction sustained for $60 \mathrm{~s}$ and (4) repeated contractions. These measures have been validated as a measure of activation of the individual pelvic floor muscles. ${ }^{20}$

Other secondary outcomes that will be measured as outlined in table 1 are:

1. International Continence Society Male Short Form (incontinence subscale) (ICS-Male SF): Measures the symptomatology and 'bothersomeness' of incontinence for men with prostatic disease.

2. Self-assessed 24-hour pad weight test: So that the test can be completed at home, men will be provided with a digital scale to weigh all pads used in a 24-hour period. The start and end of the test will be prompted using the SMS service, and a bladder diary will be collected for this period. The measure of pad weight (grams) is recorded as the secondary outcome.

3. International Physical Activity Questionnaire: Used to assess physical activity over the same period as the 24hour pad weight test.

4. 12-Item Short Form Survey (SF-12) ${ }^{27}$ : Used to measure health-related quality of life based on the recommendations of a recent systematic review of quality-of-life measures for prostate cancer. ${ }^{28}$ The Short-Form Six-Dimension (SF-6D) computation may also be applied to SF-12 data to compute the utility weights required to construct Quality-Adjusted Life-Year (QALY) measures for use in the cost-effectiveness analysis.

5. EuroQol Five Dimension Five Level (EQ-5D-5L) ${ }^{29}$ : Used to calculate the QALY saved for cost-effectiveness analysis.

6. Incontinence-related costs (use of health services/devices): Recorded prospectively for every participant for 12 months. Men will be prompted to input data using the data collection system. Data will include visits to healthcare practitioners (eg, therapists, general practitioner), drugs and number of devices such as pads or bed/chair protectors used.

7. Sexual function: Determined using the question 'Are you currently able to achieve a full erection?'.

8. Bowel function: Determined using questions previously described in a clinical trial of postprostatectomy incontinence. $^{30}$

\section{Data integrity}

All data will be directly collected into the REDCap program. Any inconsistencies in the data will be explored and resolved. The database will be backed-up regularly on 
Table 2 Trial registration data

\begin{tabular}{|c|c|}
\hline Data category & Information \\
\hline $\begin{array}{l}\text { Primary registry and trial identifying } \\
\text { number }\end{array}$ & Australia New Zealand Clinical Trials Registry [ACTRN12617000788370] \\
\hline Date of registration in primary registry & $30 / 05 / 2017$ \\
\hline Secondary identifying numbers & Universal Trial Number U1111-1196-7696 \\
\hline Sources of monetary or material & Sponsors (below) \\
\hline
\end{tabular}

support

\begin{tabular}{|c|c|}
\hline Primary sponsor & $\begin{array}{l}\text { National Health and Medical Research Council-Research Committee Secretariat } \\
\text { NHMRC, GPO Box 1421, Canberra, ACT } 2601\end{array}$ \\
\hline Secondary sponsor & $\begin{array}{l}\text { Queensland Health Physiotherapy Research Fellowship-Queensland Health Building } \\
\text { 147-163 Charlotte Street, Brisbane, Queensland } 4000\end{array}$ \\
\hline Contact for public queries & RS (r.stafford@uq.edu.au) \\
\hline Contact for scientific queries & RS (r.stafford @uq.edu.au) \\
\hline Public title & Personalised pelvic floor muscle training for urinary incontinence after prostatectomy \\
\hline Scientific title & $\begin{array}{l}\text { Efficacy of a personalised pelvic floor muscle training programme on urinary } \\
\text { incontinence after radical prostatectomy: randomised clinical trial with embedded } \\
\text { physiological studies }\end{array}$ \\
\hline Countries of recruitment & Australia \\
\hline Health condition or problem studied & Postprostatectomy incontinence \\
\hline Intervention & $\begin{array}{l}\text { Urethral muscle training-comprehensive individualised programme focused on } \\
\text { training the striated muscles that pressurise the urethra }\end{array}$ \\
\hline \multirow[t]{2}{*}{ Key inclusion and exclusion criteria } & $\begin{array}{l}\text { Inclusion criteria: aged } 30-70 \text { years; scheduled to undergo radical prostatectomy } \\
\text { for prostate cancer; able to attend assessment and treatment sessions; able to } \\
\text { understand English }\end{array}$ \\
\hline & $\begin{array}{l}\text { Exclusion criteria: urinary incontinence prior to surgery; previous prostate/urethral } \\
\text { surgery; assessment/training of pelvic floor muscles in preceding } 6 \text { months; scheduled } \\
\text { to undergo or had previously undergone radiation therapy for prostate cancer. }\end{array}$ \\
\hline Study type & $\begin{array}{l}\text { Randomised controlled trial, assessor and statistician blinding, automatic independent } \\
\text { randomisation }\end{array}$ \\
\hline Date of first enrolment & $27 / 07 / 2018$ \\
\hline Target sample size & 363 \\
\hline Recruitment status & Recruiting \\
\hline Primary outcome(s) & Continence defined by the 24-hour pad weight test \\
\hline Key secondary outcomes & $\begin{array}{l}\text { SF-12; ICS-Male SF Questionnaire; continence-related costs; 24-hour pad weight } \\
\text { test; measures of neuromuscular control of urethral pressure; IPAQ; EQ-5D-5L } \\
\text { questionnaire; questions related to sexual and bowel function }\end{array}$ \\
\hline
\end{tabular}

EQ-5D-5L, EuroQol 5 Dimension 5 Level; ICS-Male SF, International Continence Society Male Short Form; IPAQ, International Physical Activity Questionnaire; SF-12, 12-Item Short Form Survey.

a secure network and be compliant to the International Conference on Harmonisation (ICH) Guideline for Good Clinical Practice, ${ }^{31}$ according to our data management plan. Study personnel will only be able to access the database with a personal login and password.

\section{Retention of documents}

Study investigators will maintain adequate and accurate records to enable the conduct of the study to be fully documented and the study data to be subsequently verified. After completion of the study, study data will be archived by The University of Queensland for a minimum of 15 years.

\section{Data analysis}

Primary endpoint and sample size justification

The primary outcome is the proportion of participants continent at 3 months. This study is powered to determine whether urethral training is more efficacious than conventional training. This difference is expected to be smaller than the difference between urethral and no training. Data from seven RCTs ${ }^{32}$ indicate $\sim 60 \%$ of men receiving conventional training will be incontinent at 3 months. With 97 men per group, a reduction of incontinence by a third, from $60 \%$ to $40 \%$ of men at 3 months (conservatively based on the difference identified in a 
previous study, ${ }^{8}$ which included some features we consider critical in the proposed programme) can be detected with $80 \%$ power and a two-sided significance level of 0.05 .

Our data of healthy men suggest $\sim 55 \%$ have good baseline NM control (RE Stafford et al, unpublished data, 2018), defined as ability to achieve both $\geq 4.1 \mathrm{~mm}$ of SUS displacement and $\geq 2.4 \mathrm{~mm}$ of PR displacement as assessed with transperineal ultrasound imaging during voluntary contraction (RE Stafford et al, unpublished data, 2018). We make the assumption that among those with good baseline NM control, incontinence outcomes at 3 months will be similar regardless of treatment arm. With these assumptions and sample size, we have $88 \%$ power to detect a significant interaction between baseline NM control and treatment arm if we assume that $70 \%$ of men with good baseline NM control will be continent at 3 months regardless of treatment, but that, of the men with poor baseline NM control, $90 \%$ and $50 \%$ will be continent with urethral or conventional training, respectively. We will recruit 121 men per arm (adjusting for a potential dropout rate of $20 \%$ ). This is feasible based on a recent RCT of 308 participants ${ }^{6}$ from a subset of our referral sources.

\section{Statistical analysis}

A biostatistician (JK) will analyse blinded data, with all the patients enrolled and randomised to treatment/ no treatment arms comprising the data set for analysis. Baseline characteristics of groups will be tabulated using summary statistics. If required, multiple imputations will be used to account for missing data, with imputation conducted separately for each treatment arm.

\section{Primary analysis}

Analyses will be by intention-to-treat of all randomised participants. For the binary continence outcome at each time point, a hierarchical logistic regression model including random effects for physiotherapists, terms for treatment group and baseline control and interaction between them will be fit. The model will also include a term for the stratifying variable of the surgeon. For the primary hypothesis, this model will be interrogated to yield differences in the proportions of participants recovering continence at 3 months between the groups and $95 \% \mathrm{CIs}^{33}$ The model will be similarly interrogated to determine whether the effect of urethral training relative to conventional training is moderated by NM control at baseline. A secondary analysis will fit a longitudinal model for the multiple outcomes from each participant, including random effects for each participant as well as for physiotherapist, and a three-way interaction term between time, randomised treatment group and baseline NM control, and all 2-way interactions and main effects, as well as a term for the surgeon.

\section{Secondary analyses}

The continuous measure of continence (24-hour pad weight test) and other continuous outcomes (ICS-male
SF, SF-12, EQ-5D-5L, sexual function and bowel function) will be compared between groups by fitting similar random effects linear regression models. Time to recovery of continence will be compared between groups using a Cox proportional hazards model using the weekly self-assessed pad test. Model assumptions (linearity, normality and homoscedasticity of residuals for the linear regression models and proportional hazards) will be assessed using standard diagnostic plots.

\section{Mediation analysis}

To determine the extent to which the effect of urethral training on the primary outcome and on the continuous measurement of continence is mediated through an improvement in NM control, as hypothesised, we will apply a causal mediation analysis. ${ }^{34}$ Mediation analyses will be conducted treating the 6-week and 3-month NM control measures as the potential mediators, with all analyses adjusted for baseline NM control and other potential confounders of the outcome-mediator relationship (eg, prostate volume, Gleason score, age and so on). The analysis will be conducted in two stages: at the first stage, the effect of randomisation to the study arms on NM control measures will be assessed. In the second stage, models will be fit to estimate the direct effect of randomised group on the outcome and the indirect effect of randomised group on the outcome that acts through the putative mediator. Whether the indirect effect of treatment on the outcomes changes depending on the level of NM control achieved after surgery will be investigated through the inclusion of interaction terms between treatment group and postsurgery NM control variables in the mediation analyses.

If significant mediation is found, logistic regression will be undertaken using the 3-month data of the NM control variables to determine which variables are best linked with continence. A factor analysis across all participants will be applied to extract common NM control features from the NM control variables. NM control features will then be used as predictor variables in the logistic regression analysis to assess the relative contribution of each to the odds of regaining continence.

\section{Cost-effectiveness analysis}

Costs of services and devices will be estimated using market prices. We will undertake two analyses. First, we will compare cost-effectiveness between treatment arms (3 and 12-month data). Second, we will address the question of whether treatment is more cost-effective if only offered to men with poor NM control at baseline. For this analysis, we will test the interaction between treatment arm and baseline NM control. This will test the hypothesis that continence-related costs will be similar for men with good NM control, regardless of treatment allocation, but costs will be significantly less for men with poor baseline NM control allocated to urethral training. QALYs saved will be computed using the EQ-5D-5L data and SF-12 data, using an SF-6D algorithm. Cost-effectiveness ratios will be computed and n-way sensitivity analyses will be conducted 
to produce cost-effectiveness acceptability curves for relevant sets of assumptions about costs and outcomes.

\section{Contamination between groups}

We do not anticipate contamination between urethral training and conventional training as different therapists will apply each intervention. Further, ultrasound imaging is required for urethral training and is not available to the other groups. It is possible that men allocated to no treatment will be exposed to information regarding PFMT (in addition to that provided to them in written form at the presurgery visit to the physiotherapist) through searching the Internet and from friends and family. However, evidence from several trials shows that provision of information alone does not lead to clinical improvement. ${ }^{32}$

\section{ETHICS AND DISSEMINATION}

This study is supported by grants from the National Health and Medical Research Council of Australia and Queensland Health, is registered with the Australian New Zealand Clinical Trials Registry and has ethical approval from the Human Research Ethics Committees of The University of Queensland (2017001736), Uniting Care (UCHHREC1739) and Metro South (HREC/17/ $\mathrm{QPAH} / 591$ ) (table 2). The funders have not contributed to the design of the trial nor will they be involved in its conduct or management. The current protocol is version 2 (2 May 2018), and any future protocol modifications would require approval by the principal investigator (PWH) and formal amendment.

Potential participants will be invited to participate via their treating urologist. To manage this unequal relationship, potential participants will be informed that trial assessments and interventions are not part of their routine care, that they are free to decide to participate without coercion and that the decision to not participate will not influence their management or relationship with their urologist. Although the urologist may discuss the trial with the patient, they will not be involved in the screening or consent process nor any of the assessment or training sessions.

As standard practice at the Princess Alexandra Hospital involves provision of written information only, no treatment will be withheld from patients. If a patient continues to experience incontinence at the completion of the primary endpoint, we will provide them with information of treatment options.

Participants will be given contact details of the project manager for queries or concerns. As all treatments are low risk, no adverse events are anticipated, but if any do occur, they will be recorded and reported. Records of complaints arising from the trial will be acted on in accordance with institutional policy. Participants will be informed they are free to withdraw from the study at any time. They will be given the option to receive the results of the study in summary format at the conclusion of the trial.
Participant data sheets will be stored in a locked cabinet, in addition to electronic storage of scanned copies on the secure institutional data server. All other data will be stored in electronic format in a deidentified manner. Consent forms (that include both a code and identifiable information) will be stored separately to the coded data in a locked cabinet.

The data collected in this trial will be thoroughly analysed and published. As this data will be specific to the interventions provided, we do not anticipate any secondary use of the information. The trial consent form includes the option to opt out of making data available for future analyses.

The results will be disseminated through publication in peer-reviewed scientific journals and presented at major international scientific meetings. Further, the study outcomes will be disseminated to the broader community through paper-based and online media.

\section{Patient and public involvement}

The research question was based on hypotheses developed from basic science data and informed by the poor results reported from previous RCTs but did not involve direct patient or public involvement. The primary outcome measure was based on published data of patients' preferences. Patients did not contribute to the design of this study. Patients were not involved in the recruitment to or conduct of the study. The results will be disseminated to study participants in the form of a summary (written in lay language) at the completion of the trial. The acceptability of the nature and burden of the intervention was confirmed by application of the treatment protocol in pilot trials with patients prior to commencement of the study.

\section{Author affiliations}

${ }^{1}$ School of Health and Rehabilitation Sciences, The University of Queensland, Brisbane, Queensland, Australia

${ }^{2}$ Renal Medicine, Royal Brisbane \& Women's Hospital, Brisbane, Queensland, Australia

${ }^{3}$ Wesley Urology Clinic, Wesley Hospital, Brisbane, Queensland, Australia ${ }^{4}$ Epidemiology and Preventive Medicine, Monash University, Melbourne, Australia ${ }^{5}$ Department of Mechanical Engineering, University of Michigan, Ann Arbor, Michigan, USA

${ }^{6}$ Centre for the Business and Economics of Health, University of Queensland, Brisbane, Queensland, Australia

${ }^{7}$ Poche Centre for Indigenous Health, University of Queensland, Brisbane, Queensland, Australia

Contributors PH, RS, GDC, JA-M, APC and LMH conceived the study. PH, RS, GDC, JK, JA-M, APC, LC and LMH developed the experimental design for the study. JK designed the statistical analysis of the study and undertook power calculation. LC designed the health economic data collection and analysis. PH, RS, GDC, JA-M, APC and LC obtained funding for the study. All authors contributed to the preparation of the protocol manuscript, and all authors approved the final version.

Funding This clinical trial is supported by the National Health and Medical Research Council (NHMRC) of Australia (APP1146267) and a Queensland Health Physiotherapy Research Fellowship grant. PH is supported by an NHMRC Senior Principal Research Fellowship (APP1102905).

Competing interests None declared.

Patient consent for publication Not required. 
Provenance and peer review Not commissioned; externally peer reviewed.

Open access This is an open access article distributed in accordance with the Creative Commons Attribution Non Commercial (CC BY-NC 4.0) license, which permits others to distribute, remix, adapt, build upon this work non-commercially, and license their derivative works on different terms, provided the original work is properly cited, appropriate credit is given, any changes made indicated, and the use is non-commercial. See: http://creativecommons.org/licenses/by-nc/4.0/.

\section{REFERENCES}

1. AlHW. Cancer in Australia 2017. Cancer series no101. Canberra: AlHW, 2017.

2. Litwin MS, Melmed GY, Nakazon T. Life after radical prostatectomy: a longitudinal study. J Urol 2001;166:587-92.

3. Kao TC, Cruess DF, Garner D, et al. Multicenter patient self-reporting questionnaire on impotence, incontinence and stricture after radical prostatectomy. J Urol 2000;163:858-64.

4. Fowler FJ, Barry MJ, Lu-Yao G, et al. Effect of radical prostatectomy for prostate cancer on patient quality of life: results from a Medicare survey. Urology 1995;45:1007-15.

5. Katz G, Rodriguez R. Changes in continence and health-related quality of life after curative treatment and watchful waiting of prostate cancer. Urology 2007;69:1157-60.

6. Yaxley JW, Coughlin GD, Chambers SK, et al. Robot-assisted laparoscopic prostatectomy versus open radical retropubic prostatectomy: early outcomes from a randomised controlled phase 3 study. Lancet 2016;388:1057-66.

7. Dumoulin C, Hay-Smith J. Pelvic floor muscle training versus no treatment, or inactive control treatments, for urinary incontinence in women. Cochrane Database Syst Rev 2010;1:CD005654.

8. Van Kampen M, De Weerdt W, Van Poppel H, et al. Effect of pelvic-floor re-education on duration and degree of incontinence after radical prostatectomy: a randomised controlled trial. Lancet 2000;355:98-102.

9. Campbell SE, Glazener CM, Hunter KF, et al. Conservative management for postprostatectomy urinary incontinence. Cochrane Database Syst Rev 2012;1:CD001843.

10. Stafford RE, Ashton-Miller JA, Constantinou CE, et al. A new method to quantify male pelvic floor displacement from 2D transperineal ultrasound images. Urology 2013;81:685-9.

11. Stafford RE, van den Hoorn W, Coughlin G, et al. Postprostatectomy incontinence is related to pelvic floor displacements observed with trans-perineal ultrasound imaging. Neurourol Urodyn 2018;37:658-65.

12. Stafford RE, Sapsford R, Ashton-Miller J, et al. A novel transurethral surface electrode to record male striated urethral sphincter electromyographic activity. J Urol 2010;183:378-85.

13. Dorey G, Glazener C, Buckley B, et al. Developing a pelvic floor muscle training regimen for use in a trial intervention. Physiotherapy 2009;95:199-208.

14. Stafford RE, Ashton-Miller JA, Constantinou CE, et al. Novel insight into the dynamics of male pelvic floor contractions through transperineal ultrasound imaging. J Urol 2012;188:1224-30.

15. Stafford RE, Mazzone S, Ashton-Miller JA, et al. Dynamics of male pelvic floor muscle contraction observed with transperineal ultrasound imaging differ between voluntary and evoked coughs. $J$ Appl Physiol 2014;116:953-60.

16. Desautel MG, Kapoor R, Badlani GH. Sphincteric incontinence: the primary cause of post-prostatectomy incontinence in patients with prostate cancer. Neurourol Urodyn 1997;16:153-60.

17. Presti JC, Schmidt RA, Narayan PA, et al. Pathophysiology of urinary incontinence after radical prostatectomy. J Urol 1990;143:975-8.

18. Groutz A, Blaivas JG, Chaikin DC, et al. The pathophysiology of postradical prostatectomy incontinence: a clinical and video urodynamic study. J Urol 2000;163:1767-70.

19. Hall LM, Aljuraifani R, Hodges PW. Design of programs to train pelvic floor muscles in men with urinary dysfunction: Systematic review. Neurourol Urodyn 2018;37:2053-87.

20. Stafford RE, Coughlin G, Lutton NJ, et al. Validity of estimation of pelvic floor muscle activity from transperineal ultrasound imaging in men. PLoS One 2015;10:e0144342.

21. Chan AW, Tetzlaff JM, Altman DG, et al. SPIRIT 2013 statement: defining standard protocol items for clinical trials. Ann Intern Med 2013;158:200-7.

22. Slade SC, Dionne CE, Underwood M, et al. Consensus on Exercise Reporting Template (CERT): explanation and elaboration statement. Br J Sports Med 2016;50:1428-37.

23. Hall M, Hinman RS, Wrigley TV, et al. The effects of neuromuscular exercise on medial knee joint load post-arthroscopic partial medial meniscectomy: 'SCOPEX', a randomised control trial protocol. BMC Musculoskelet Disord 2012;13:233.

24. Glazener C, Boachie C, Buckley B, et al. Urinary incontinence in men after formal one-to-one pelvic-floor muscle training following radical prostatectomy or transurethral resection of the prostate (MAPS): two parallel randomised controlled trials. Lancet 2011;378:328-37.

25. Cooperberg MR, Master VA, Carroll PR. Health related quality of life significance of single pad urinary incontinence following radical prostatectomy. J Urol 2003;170(2 Pt 1):512-5.

26. Kirschner-Hermanns R, Jakse G. Quality of life following radical prostatectomy. Crit Rev Oncol Hematol 2002;43:141-51.

27. Ware J, Kosinski M, Keller SD. A 12-Item Short-Form Health Survey: construction of scales and preliminary tests of reliability and validity. Med Care 1996;34:220-33.

28. Hamoen EH, De Rooij M, Witjes JA, et al. Measuring health-related quality of life in men with prostate cancer: a systematic review of the most used questionnaires and their validity. Urol Oncol 2015;33:69. e19-69.e28.

29. Herdman M, Gudex C, Lloyd A, et al. Development and preliminary testing of the new five-level version of EQ-5D (EQ-5D-5L). Qual Life Res 2011;20:1727-36.

30. Glazener C, Boachie C, Buckley B, et al. Conservative treatment for urinary incontinence in Men After Prostate Surgery (MAPS): two parallel randomised controlled trials. Health Technol Assess 2011;15:1-290.

31. ICH. ICH guideline for good clinical practice, 2016.

32. Anderson CA, Omar MI, Campbell SE, et al. Conservative management for postprostatectomy urinary incontinence. Cochrane Database Syst Rev 2015;1:CD001843.

33. Muller CJ, MacLehose RF. Estimating predicted probabilities from logistic regression: different methods correspond to different target populations. Int J Epidemiol 2014;43:962-70.

34. Emsley R, Dunn G, White IR. Mediation and moderation of treatment effects in randomised controlled trials of complex interventions. Stat Methods Med Res 2010;19:237-70. 